\title{
Impact of harmonic distortion on the supraharmonic emission of pulse- width modulated single-phase power electronic devices
}

\author{
E. Kaufhold ${ }^{1}$, J. Meyer ${ }^{1}$, P. Schegner ${ }^{1}$ \\ ${ }^{1}$ Institute of Electrical Power Systems and High Voltage Engineering \\ Technische Universitaet Dresden \\ Dresden, Germany \\ e-mail: elias.kaufhold@tu-dresden.de
}

\begin{abstract}
This study demonstrates the frequency coupling in pulse width modulated (PWM) single-phase converters and inverters. As current state of the art, the harmonic frequency range, e.g. from fundamental frequency up to $2 \mathrm{kHz}$, is typically assessed separately from the so-called supraharmonic frequency range, i.e. above $2 \mathrm{kHz}$ up to $150 \mathrm{kHz}$. The frequency coupling between the harmonic and supraharmonic frequency range has not been thoroughly studied and is currently often neglected in the design process as well as for the analysis of the emission of power electronic devices.

The aim of this study is to analyse the behaviour of the frequency coupling between the harmonic and the supraharmonic range. In addition, laboratory measurements of a commercially available single-phase inverter for photovoltaic applications are shown to verify the findings.
\end{abstract}

Key words. Converters, Harmonics, Power Electronics, Power Quality, Supraharmonics.

\section{Nomenclature}

\begin{tabular}{cc}
\hline Symbol & Definition \\
\hline$x$ & Real value \\
$\underline{x}$ & Complex value \\
$\hat{x}$ & Amplitude value \\
$y(x)$ & Dependency of $y$ on $x$ \\
$X$ & Root mean square (RMS) value / \\
$|x|$ & magnitude \\
$\boldsymbol{x}$ & Column vector \\
$\boldsymbol{X}$ & Matrix \\
$y_{v \mu}$ & Complex matrix element with the \\
& indices $v$ and $\mu$
\end{tabular}

\section{Introduction}

With the demand for a higher energy efficiency and with the aim to meet the climate goals, the number of power electronic (PE) devices increases in low voltage (LV) networks. Modern loads based on topologies with semiconductor switches replace traditional loads. In this study, PE devices are defined according to their power conversion into converters (AC-AC or DC-DC conversion), inverters (DC-AC conversion) and rectifiers (AC-DC conversion). These devices are integrated into different applications, e.g. electric vehicle (EV) chargers, battery storage systems (BSS) and photovoltaic (PV) systems. The PE devices contain different components, e.g. the control, the switches, filters etc. while this study focusses specifically on converters and inverters.

The non-ideal behaviour of PE devices leads to an interaction with the connected power grid, i.e. in this study the LV network. These interactions can be studied in terms of the voltage and the current at the Point of Connection (PoC) of a grid-connected PE device. In the past, an increasing number of problems in terms of harmonic instabilities and high distortion levels has been measured ([1], [2]) that caused the shutdown of PE devices [3]. The first occurrence of such harmonic instabilities has been reported in the Swiss railway grid [4]. Consequently, the topic of current emission limits and suitable assessment, modelling and analysis strategies has become a growing research field [5]. These studies have included the steady state behaviour, stability analysis [6], e.g. more recently by probabilistic means [7], but also transients in the supply voltage ([8], [9]).

The frequency ranges, e.g. the harmonic frequency range for frequencies above fundamental frequency up to $2 \mathrm{kHz}$ and the subharmonic frequency range below fundamental frequency are often studied and modelled separately from the supraharmonic frequency range [10], i.e. above $2 \mathrm{kHz}$. In [11], qualitative measurements have been performed to analyse different impact factors on the supraharmonic frequency range, i.e. the DC-link voltage, the device power level, the AC voltage in terms of its fundamental frequency and specific harmonic waveform distortions. An impact of the harmonic distortion of the supply voltage on the supraharmonic emission has been found, but not further studied with regard to their origin and interaction mechanism. On the other hand, analytic studies are currently still not considering the impact of subharmonic or harmonic distortion components on the supraharmonic emission of PE devices, i.e. the switching frequency emission.

Consequently, this paper addresses the frequency coupling between the harmonic and the supraharmonic frequency range and evaluates the significance of the impact of the 
harmonic distortion on the supraharmonic emission. It is structured as follows: Section 2 briefly explains the state of the art. Section 3 studies the frequency coupling in theory by considering the voltage distortion at the switches. Section 4 presents laboratory measurements that have been performed on a commercially available single-phase inverter for PV applications and discusses briefly the results. Finally, Section 5 concludes the work.

\section{State of the art}

\section{A. System model}

The system model can be separated into two main parts, namely the application side and the network side. The application can be a PV system consisting of a PV inverter with respective PV modules as exemplarily depicted in Figure 1, but also a BSS or an EV charger. While even further applications are possible, these three applications are the most common when considering LV networks which rely on similar technical operation principles. The second part of the system model is the LV network to which the devices are connected.

\section{1) Low voltage network}

The LV network is typically represented as an $R L$ equivalent. This simplification is often found in practice, e.g. for controller and filter design methods of manufacturers, while other studies, especially in terms of stability analyses, have shown the impact of resonances on the device stability [12]. The challenge for all these studies is the large variation of the network impedance characteristic [13] with regard to generic assumptions. It can be concluded that the connected devices and the network topology will affect the network impedance characteristic significantly.

\section{2) Single-phase converters and inverters}

The topologies of single-phase converters and inverters are mainly similar in their structure. However, the design of individual components such as the control, e.g. $P I$ or $P R$ controllers, the filter design, e.g. $L C$ - or $L C L$-filters or even more advanced implementations, can vary significantly, e.g. [14]. This will reflect in the amplitude of the harmonic distortion of the grid-side current $i_{\mathrm{AC}}(t)$ as well as stability issues. The different design approaches strongly depend on the manufacturer, whereby the manufacturers typically do not disclose their device designs with the respective topologies and parameters. This is why different modelling approaches have been developed to allow studies from the different perspectives, e.g. from the manufacturer side and the network operator side, e.g. the distribution system operator (DSO).

\section{B. Modelling approaches}

According to the available knowledge, the different modelling approaches can be separated into the white-box, the grey-box and the black-box approach.

\section{1) White-box approach}

The highest degree of knowledge is demanded for the white-box approach. All components are represented in detail based on known parameters. To overcome the effort that has to be made to model the different component designs, a modular modelling approach has been proposed [15]. However, not only the implementation effort is large, but also the computational requirements for solving the simulations are high, so that appropriate solver settings are essential, e.g. [16].

For a simplified analysis of converters and inverters, a small signal model in terms of a linear time-periodic (LTP) characteristic is often derived that can be represented in its most general form with

$$
\begin{aligned}
& \dot{\boldsymbol{x}}(t)=\boldsymbol{A}(t) \boldsymbol{x}(t)+\boldsymbol{B}(t) \boldsymbol{u}(t) \\
& \boldsymbol{y}(t)=\boldsymbol{C}(\mathrm{t}) \boldsymbol{x}(t)+\boldsymbol{D}(t) \boldsymbol{u}(t)
\end{aligned}
$$

for the real value matrices $\boldsymbol{A}(t), \boldsymbol{B}(t), \boldsymbol{C}(t)$ and $\boldsymbol{D}(t)$, the input vector $\boldsymbol{u}(t)$, the state space vector $\boldsymbol{x}(t)$ and the output vector $\boldsymbol{y}(t)$ with the appropriate dimensions as introduced in [17]. Transferring this time domain representation into the harmonic domain leads to the well-known harmonic transfer functions (HTFs). By Fourier expansion, the set of harmonic transfer functions can be represented with the matrix

$$
\boldsymbol{H}=\left[\begin{array}{ccccc}
\ddots & \vdots & \vdots & \vdots & \\
\ldots & H_{0}(s-j \omega) & H_{-1}(s) & H_{-2}(s+j \omega) & \ldots \\
\ldots & H_{1}(s-j \omega) & H_{0}(s) & H_{-1}(s+j \omega) & \ldots \\
\ldots & H_{2}(s-j \omega) & H_{1}(s) & H_{0}(s+j \omega) & \ldots \\
& \vdots & \vdots & \vdots & \ddots
\end{array}\right] .
$$

Due to the resulting infinite number of elements of this matrix, a truncation to an appropriate dimension is typically applied. However, this truncation reduces the frequency range of considerable frequency coupling

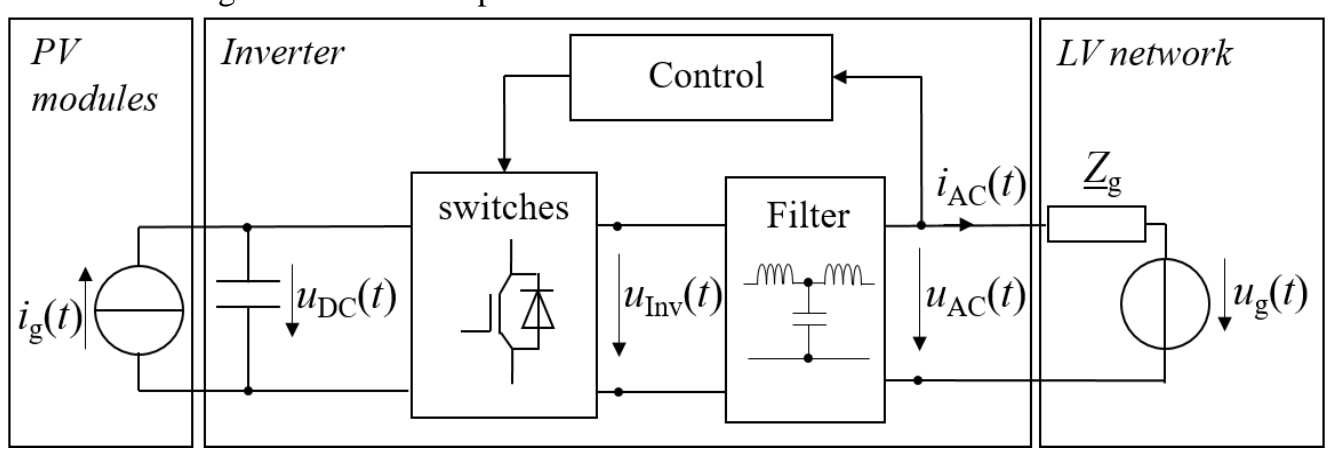

Figure 1: Scheme of a PV inverter in the low voltage network 
components, i.e. the non-diagonal elements in (2). The truncation is typically chosen to reduce the model to the harmonic range up to $2 \mathrm{kHz}$, which is sufficient for studies of control interactions with the power grid by means of control theory or harmonic power flow studies. The interaction within a larger frequency range has not been analysed.

\section{2) Grey-box approach}

As a second modelling approach, the grey-box approach includes partial knowledge into the model. Often, parameters of a known topology are estimated, e.g. with a Kalman filter [18] or more recently with Neural Networks [19], but could also include the identification of the topology of device components itself. Due to the lack of identification methods, grey-box models have not received a lot of attraction in terms of modelling PE devices.

\section{3) Black-box approach}

Black-box models require the lowest degree of knowledge. Only generic assumptions are made, e.g. with respect to a device class, while all parameters and topologies or topology equivalents in terms of general representations have to be identified by measurements. Black-box models are used for network studies, e.g. for harmonic power flow calculations. While alternative model representations study the use of nonlinear structures, e.g. in terms of the Hammerstein model [20], the Hammerstein-Wiener model [21], [22] or Neural Networks [23], the conventional representation makes use of classical circuit elements in terms of the decoupled Norton model, i.e. a current source and an admittance. While simple (de-coupled) non-linear models do not address the accurate representation of frequency coupling components, the more advanced, coupled Norton model allows to represent the frequency couplings e.g. by adding multiple voltage-controlled current sources to the de-coupled Norton model.

\section{Interactions between harmonic and supraharmonic range}

In the context of power supply, frequency coupling implies that a single frequency component in the supply voltage $u_{\mathrm{AC}}(t)$ at the PoC causes a multi-frequent current $i_{\mathrm{AC}}(t)$ at the PoC. From a black-box perspective, these couplings can be identified according to the principle of a frequency sweep [17]. If with respect to a chosen reference voltage $u_{\text {ref }}(t)$ only one frequency component at harmonic order $\mu$ is changed (magnitude and/or phase angle), the changes in the current frequency components at order $v$ can be measured and the elements $y_{v \mu}$ with

$$
\begin{aligned}
& y_{v \mu}=\frac{\underline{I}_{i v}-\underline{I}_{\text {ref } v}}{\underline{U}_{i \mu}-\underline{U}_{\text {ref } \mu}} \\
& u_{\mathrm{Inv}}(t)=\frac{4 \bar{u}_{\mathrm{DC}}}{\pi} \sum_{n=1}^{\infty} \frac{J_{n}\left(n \frac{f_{1} \pi}{f_{\mathrm{car} 2} m_{\mathrm{a}}}\right)}{n \frac{2 \pi f_{1}}{2 \pi f_{\mathrm{car}}}} \sin \left(n \frac{\pi}{2}\right) \cos \left(2 n \pi f_{1} t\right) \\
& +\frac{4 U_{\mathrm{DC}}}{\pi} \sum_{m=1}^{\infty} \sum_{n=-\infty}^{\infty} \frac{1}{q} J_{2 n-1}\left(q \frac{\pi}{2} m_{\mathrm{a}}\right) \cos ([m+1-n] \pi) \cos \left(4 m \pi f_{\mathrm{car}} t+[2 n-1] 2 \pi f_{1} t\right)
\end{aligned}
$$

can be calculated to obtain the frequency coupling matrix (FCM) $\boldsymbol{Y}$ of the measured device. However, the identification of frequency coupling matrices is usually limited to the harmonic range and consequently possible effects in the supraharmonic frequency range have not been studied yet.

For pulse-width modulation (PWM) based converters and inverters, the voltage spectrum of $u_{\text {Inv }}(t)$ at the switches has been derived for an asymmetric, regular sampled unipolar PWM. While in theory, natural modulation is often considered, the spectrum of $u_{\mathrm{Inv}}(t)$ of real systems, i.e. the spectrum of the asymmetric, regular sampled modulation, contains further frequency components. An analytic description of $u_{\text {Inv }}(t)$ is shown in (8) according to [24] with the averaged DC-link voltage $\bar{u}_{\mathrm{DC}}$, the carrier frequency of the modulation $f_{\text {car }}$, the fundamental frequency of the gridside voltage $f_{1}$, the Bessel functions $J_{n}$ of first kind at order $n$, the modulation index $m_{\mathrm{a}}$ with

$$
m_{\mathrm{a}}=\frac{U_{\mathrm{AC}}\left(f_{1}\right)}{\bar{u}_{\mathrm{DC}}}
$$

the parameter $q$ in terms of

$$
q=2 m+(2 n-1) \frac{f_{1}}{f_{\mathrm{car}}}
$$

and the counters $n$ and $m$. According to (8), the modulated voltage at the semiconductor switches, i.e. the bridge, is a multiplication of the DC-link voltage and the reference signal in time domain and consequently a convolution in frequency domain. However, the derivation of (8) implies two simplifying assumptions, which are discussed in the next subsections.

\section{A. DC-link voltage ripple}

While the DC-link voltage ripple is considered for the design of the DC-link capacitor, for the calculation of the output voltage of the bridge in (8) it is assumed constant, which still represents the most recent analysis standard.

To include the DC voltage ripple, it should be noted, that the power equilibrium between the power at the DC-link $p_{\mathrm{DC}}$ and the grid-side AC-power $p_{\mathrm{AC}}$ leads to

$$
p_{\mathrm{DC}}(t) \approx p_{\mathrm{AC}}(t)=\hat{u}_{\mathrm{AC}} \cos \left(2 \pi f_{1} t\right) \hat{\imath}_{\mathrm{AC}} \cos \left(2 \pi f_{1} t\right)
$$

and consequently

$$
p_{\mathrm{DC}}(t) \approx p_{\mathrm{AC}}(t)=\frac{\hat{u}_{\mathrm{AC}} \hat{\imath}_{\mathrm{AC}}}{2}+\frac{\hat{u}_{\mathrm{AC}} \hat{\imath}_{\mathrm{AC}}}{2} \cos \left(4 \pi f_{1} t\right)
$$

if the internal losses are neglected by taking into account the very high efficiencies of modern converters and inverters.

A power factor of one is assumed for reasons of 
the DC-link voltage $u_{\mathrm{DC}}(t)$ can be calculated for a DC-link capacitor with the capacity $C_{\mathrm{DC}}$ in terms of

$$
\hat{u}_{D C \text { rip }}=\frac{\bar{p}_{\mathrm{AC}}}{2 \pi f_{1} \bar{u}_{\mathrm{DC}} C_{\mathrm{DC}}}
$$

for

$$
p_{\mathrm{AC}}(t)=\bar{p}_{\mathrm{AC}}+p_{\mathrm{AC}}(t)
$$

and

$$
u_{\mathrm{DC}}(t)=\bar{u}_{\mathrm{DC}}+\hat{u}_{D C \text { rip }} \sin \left(4 \pi f_{1} t\right)
$$

Consequently, $\hat{u}_{D C \text { rip }}$ will introduce further frequency components in the spectrum of $u_{\mathrm{Inv}}(t)$ compared to (8).

\section{B. Harmonic distortion of supply voltage}

Furthermore, (8) only takes into account a non-distorted supply voltage at the PoC. However, the interaction between the power grid and the PE device itself will lead to a voltage distortion at the $\mathrm{PoC}$ as soon as the network impedance is nonzero, which is always the case in real networks. Additionally, the background distortion that results from the interaction of all other devices with the power grid will also affect the voltage waveform at the PoC, mainly at harmonics.

As a consequence, not only the fundamental frequency $f_{1}$ as shown in (8), but also the harmonic distortion of the voltage at the PoC affects the voltage distortion in the supraharmonic range, e.g. around multiples of the switching frequency. Due to the multiplication of the distortion with the switching frequency, new frequency components will result in the voltage spectrum of $u_{\text {Inv }}(t)$ with a frequency difference to the switching frequency that relates directly to the frequencies of the harmonic distortion.

\section{Laboratory test measurements}

In the following, measurements are presented to show, that the frequency coupling described in section 3 is not only theoretically existent, but also physically measureable.

\section{A. Test stand}

For the measurements, a test stand is used that consists of a programmable amplifier simulating a network. Though no additional impedance is intended between the device and the amplifier, the amplifier itself has a non-ideal impedance characteristic, i.e. the input impedance $\underline{Z}_{t}$ is not zero (see Figure 2).

As device under test, a commercially available singlephase inverter for photovoltaic application purposes has been measured. The voltage $u_{\text {Inv }}(t)$ at the inverter bridge as well as the voltage $u_{\mathrm{AC}}(t)$ and the current $i_{\mathrm{AC}}(t)$ at the gridside clamps of the inverter are measured according to Figure 1. To access the measuring points on the circuit board, i.e. to measure $u_{\mathrm{Inv}}(t)$, the measurements have been performed with an open case.

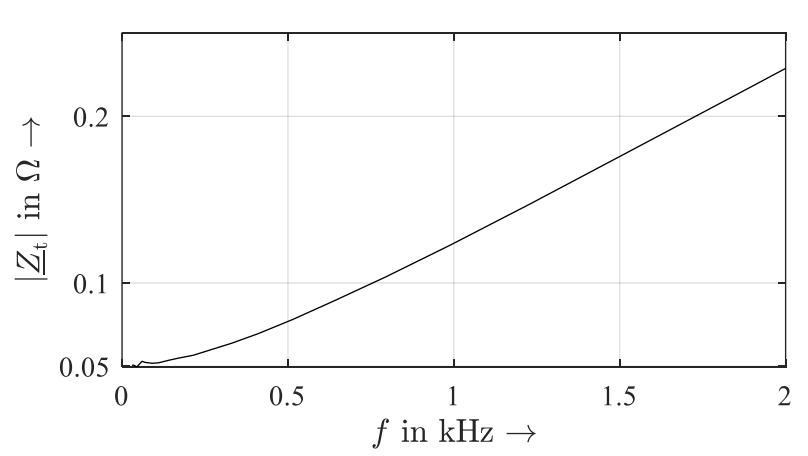

(a)

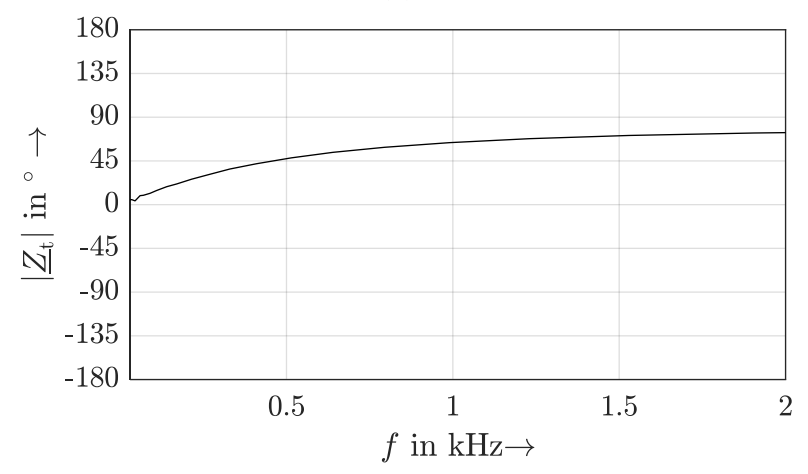

(b)

Figure 2: Magnitude of input impedance characteristic (a) and phase angle characterisitc (b) of test stand

The sample rate of the measurement equipment is $1 \mathrm{MHz}$. The measurement uncertainty is better than $10 \%$ for voltage measurements above $50 \mathrm{mV}$ and for current measurements above $15 \mathrm{~mA}$.

\section{B. Test signal}

As test signal, a frequency sweep with single-frequent voltage distortions in $10 \mathrm{~Hz}$ steps from $60 \mathrm{~Hz}$ up to $2 \mathrm{kHz}$ at a phase angle of $0^{\circ}$ and an amplitude of $2 \sqrt{2} \mathrm{~V}$ is superimposed to a fundamental voltage at $50 \mathrm{~Hz}$ and an amplitude of $230 \sqrt{2} \mathrm{~V}$. The excited frequencies are depicted for each individual measurement number in Figure 3.

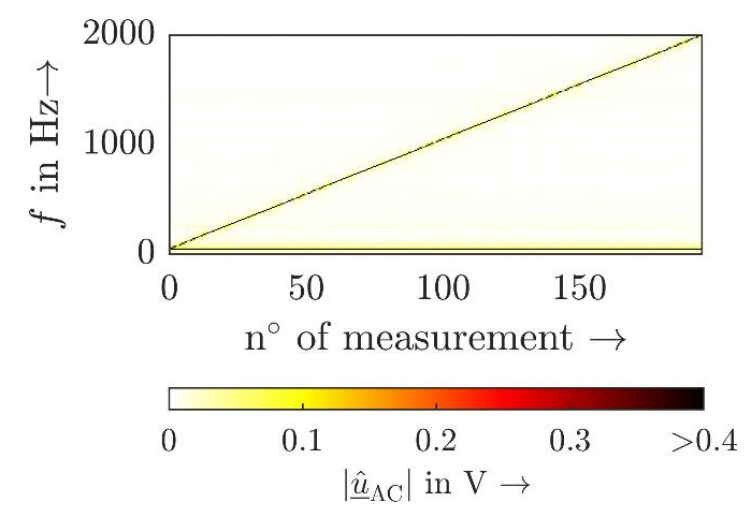

Figure 3: Frequency spectra of $u_{\mathrm{AC}}(t)$ for the applied frequency sweep 


\section{Measurement results}

With regard to the signal flow, the control and the modulator of converters and inverters define the switching signal. The voltage at the inverter bridge is exemplarily shown in Figure 4 that clearly shows the pulsed signal characteristic, where the DC ripple is visible as derived in (9).

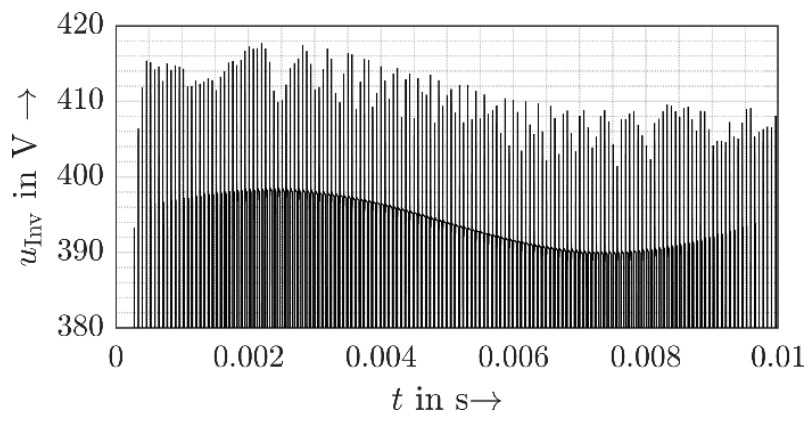

Figure 4: Detail of switched voltage $u_{\operatorname{Inv}}(t)$

Furthermore, in Figure 5, it can be seen, that the excited frequency components at the grid-side are also present in the bridge-side voltage $u_{\text {Inv }}(t)$.

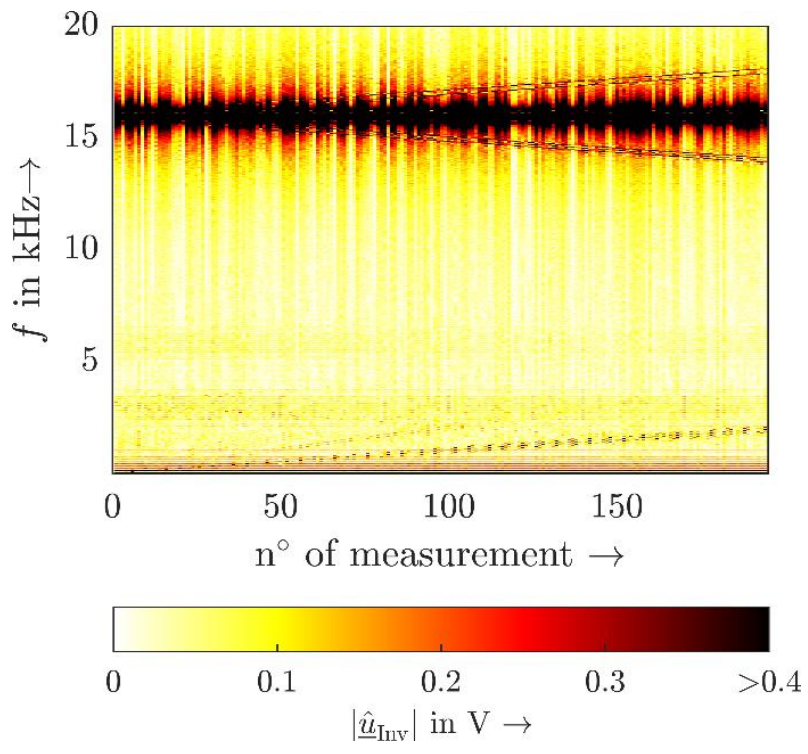

Figure 5: Frequency spectra of $u_{\text {Inv }}(t)$ for the applied frequency sweep

Consequently, the control does not suppress an unwanted distortion that is present at the inverter terminals. However, as this is a known issue, it is more interesting, that the introduced frequency coupling of the grid-side distortion, i.e. in $u_{\mathrm{AC}}(t)$, is also measurable and shows the expected characteristic. The multiplication in time domain of the distortion in $u_{\mathrm{AC}}(t)$ with the switching of the inverter bridge is clearly visible in Figure 5 as those frequency components, that go off from the switching frequency with the increasing number of measurement.

\section{Discussion}

The measurements clearly show the frequency coupling of low frequency components (harmonics) into the supraharmonic range of $u_{\mathrm{Inv}}(t)$. Strong low-frequent distortion components will consequently lead to a change in the switching frequency emission at the inverter bridge, which also proofs the observations in [11]. With an increasing number of modern self-commutating PE devices in the power grid, it is expected, that this frequency coupling effect will become more present and consequently should be considered in an early stage of the design process of PE devices for LV network applications.

When analysing the grid-side current $i_{\mathrm{AC}}(t)$, it can be stated, that for sufficiently small distortions, e.g. $2 \mathrm{~V}$, the grid-side filter circuit is able to suppress the supraharmonic components introduced by the frequency coupling, as shown in Figure 6.

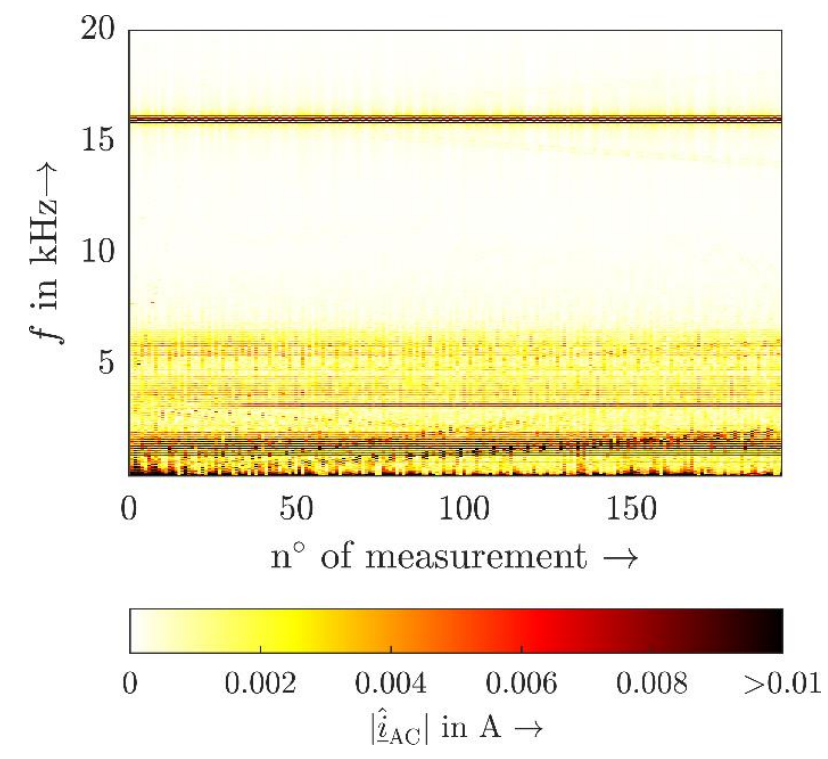

Figure 6: Frequency spectra of $i_{\mathrm{AC}}(t)$ for the applied frequency sweep

\section{Conclusion}

The paper studies the impact of the harmonic distortion in the grid-side voltage on the supraharmonic emission of PWM-based PE devices. The signal multiplication at the inverter bridge leads to a coupling of the harmonic components with the switching frequency of the device introducing additional emission in the supraharmonic range. The theoretical expectations are confirmed in laboratory measurements. A frequency sweep in the harmonic range demonstrates the presence of the expected distortion components in the supraharmonic frequency range.

As long as the additional voltage distortion can be efficiently dampened by the grid-side filter circuit, e.g. in case the voltage distortion at the Point of Connection is small enough, the demonstrated effect seems to be only minor issue for the interaction of devices with the low voltage network.

\section{Acknowledgement}

This work was funded by the Deutsche Forschungsgemeinschaft (DFG, German Research Foundation) - 360497354 . 


\section{References}

[1] J. H. R. Enslin and P. J. M. Heskes, 'Harmonic Interaction Between a Large Number of Distributed Power Inverters and the Distribution Network', IEEE Transactions on Power Electronics, vol. 19, no. 6. Institute of Electrical and Electronics Engineers (IEEE), pp. 1586-1593, Nov. 2004.

[2] M. Moghbel et al., 'Fluctuations in the Output Power of Photovoltaic Systems Distributed Across a Town with an Isolated Power System Using High-Resolution Data', 2019

[3] M. Höckel, A. Gut, M. Arnal, R. Schild, P. Steinmann, and S. Schori, 'Measurement of voltage instabilities caused by inverters in weak grids', CIRED - Open Access Proceedings Journal, vol. 2017, no. 1. pp. 770-774, Oct. 2017.

[4] E. Möllerstedt and B. Bernhardsson, 'Out of control because of harmonics-an analysis of the harmonic response of an inverter locomotive', IEEE Control Systems, vol. 20, no. 4. pp. 70-81, Aug. 2000.

[5] X. Wang and F. Blaabjerg, 'Harmonic Stability in Power Electronic Based Power Systems: Concept, Modeling, and Analysis', IEEE Transactions on Smart Grid. Institute of Electrical and Electronics Engineers (IEEE), p. 1, 2018.

[6] J. Sun, 'Impedance-based stability criterion for grid-connected inverters', IEEE Transactions on Power Electronics, vol. 26, no. 11. Institute of Electrical and Electronics Engineers (IEEE), pp. 3075-3078, Nov. 2011.

[7] E. Kaufhold, J. Meyer, S. Muller, and P. Schegner, 'Probabilistic Stability Analysis for Commercial Low Power Inverters Based on Measured Grid Impedances', in 2019 9th International Conference on Power and Energy Systems (ICPES), Dec. 2019, pp. 1-6.

[8] E. Kaufhold, J. Meyer, and P. Schegner, 'Measurement Framework for Analysis of Dynamic Behavior of Single-Phase Power Electronic Devices', in International Conference on Renewable Energies and Power Quality (ICREPQ'20), 2020.

[9] E. Kaufhold, J. Meyer, and P. Schegner, 'Transient response of single-phase photovoltaic inverters to step changes in supply voltage distortion', in 19th International Conference on Harmonics and Quality of Power (ICHQP), 2020.

[10] M. Klatt, R. Stiegler, J. Meyer, and P. Schegner, 'Generic frequency-domain model for the emission of PWM-based power converters in the frequency range from 2 to $150 \mathrm{kHz}$, IET Generation, Transmission \& Distribution, vol. 13, no. 24. pp. 5478-5486, 2019.

[11] M. Klatt, J. Meyer, P. Schegner, and C. Lakenbrink, 'Characterization of supraharmonic emission caused by small photovoltaic inverters', in Mediterranean Conference on Power Generation, Transmission, Distribution and Energy Conversion (MedPower 2016), 2016, pp. 78 (6 .)-78 (6 .).

[12] E. Kaufhold, J. Meyer, and P. Schegner, 'Impact of grid impedance and their resonance on the stability of single-phase PV-inverters in low voltage grids', in 29th IEEE International Symposium on Industrial Electronics, 2020.

[13] R. Stiegler, J. Meyer, S. Schori, M. Höckel, J. Drápela, and T. Hanzlík, 'Survey of network impedance in the frequency range 2-9 kHZ in public low voltage networks in AT / CH / CZ / GE', in 25th International Conference on Electricity Distribution, 2019, no. June, pp. 3-6.

[14] R. Teodorescu, M. Liserre, and P. Rodríguez, Grid Converters for Photovoltaic and Wind Power Systems. Chichester, UK: John Wiley \& Sons, Ltd, 2011.

[15] E. Kaufhold, J. Meyer, and P. Schegner, 'Modular White-Box Model of single-phase Photovoltaic Systems for Harmonic Studies', in 2019 IEEE Milan PowerTech, Jun. 2019, pp. 1-6.

[16] E. Kaufhold, J. Meyer, P. Schegner, A. S. Abdelsamad, and J. M. A. Myrzik, 'Comparison of solvers for time-domain simulations of single-phase photovoltaic systems', in 2020 International Conference on Smart Grids and Energy Systems (SGES), Nov. 2020, pp. 550-555.

[17] S. R. Hall and N. M. Wereley, 'Generalized Nyquist Stability Criterion for Linear Time Periodic Systems', 1990 American Control Conference. pp. 1518-1525, 1990.

[18] R. E. Kalman, 'A New Approach to Linear Filtering and Prediction Problems', Journal of Basic Engineering, vol. 82, no. 1. pp. 35-45, Mar. 1960.

[19] B. H. Lin, J. T. Tsai, and K. L. Lian, 'A Non-Invasive Method for Estimating Circuit and Control Parameters of Voltage Source Converters', IEEE Transactions on Circuits and
Systems I: Regular Papers, vol. 66, no. 12. IEEE, pp. 49114921, Dec. 2019.

[20] F. Alonge, F. D'Ippolito, F. M. Raimondi, and S. Tumminaro, 'Nonlinear Modeling of DC/DC Converters Using the Hammerstein's Approach', IEEE Transactions on Power Electronics, vol. 22, no. 4. Institute of Electrical and Electronics Engineers (IEEE), pp. 1210-1221, Jul. 2007.

[21] N. Patcharaprakiti et al., 'Modeling of single phase inverter of photovoltaic system using Hammerstein-Wiener nonlinear system identification', Current Applied Physics, vol. 10, no. 3. Elsevier B.V., pp. S532-S536, May 2010.

[22] A. S. Abdelsamad, J. M. A. Myrzik, E. Kaufhold, J. Meyer, and P. Schegner, 'Nonlinear identification approach for black-box modeling of voltage source converter harmonic characteristics', in 2020 IEEE Electric Power and Energy Conference EPEC 2020, 2020.

[23] E. Kaufhold, S. Grandl, J. Meyer, and P. Schegner, 'Feasibility of Black-Box Time Domain Modeling of Single-Phase Photovoltaic Inverters Using Artificial Neural Networks', Energies, vol. 14, no. 8. p. 2118, Apr. 2021.

[24] D. G. Holmes and T. A. Lipo, Pulse Width Modulation for Power Converters. IEEE, 2003. 